\title{
NDC F2 (International Unit)
}

National Cancer Institute

\section{Source}

National Cancer Institute. NDC F2 (International Unit). NCI Thesaurus. Code C155851.

A recognized billing qualifier that may be used to identify International Units as the NDC unit of measure. This is often used when describing IU/vial or micrograms. 